\title{
Síndrome de Cohen diagnosticado con hibridación genómica comparativa por microarreglos
}

\author{
Wilmar Saldarriaga-Gil', Laura Collazos-Saa², Julián Ramírez-Cheyne ${ }^{3}$
}

\section{RESUMEN}

El síndrome de Cohen (SC) es una enfermedad genética rara, con herencia autosómica recesiva. Se origina por daños en el gen VPS13B, locus 8q22-q23. El fenotipo característico consiste en discapacidad intelectual, microcefalia, facies dismórfica, anormalidades oftalmológicas, obesidad central e hipotonía. Solo se han publicado aproximadamente 150 casos, la mayoría de origen finlandés. Reportamos el caso de un preescolar con talla baja, craneosinostosis, facies dismórfica, hipotonía y retraso del desarrollo psicomotor a quien se le diagnosticó SC por medio de Hibridación Genómica Comparativa por microarregilos (HGCm), que mostró una deleción en homocigosis de 0.153 Mb en 8q22.2 incluyendo el gen VPS13B, OMIM \#216550. Con esta publicación contribuimos al conocimiento epidemiológico de un síndrome genético infrecuente, y mostramos el aporte de la HGCm al diagnóstico etiológico de pacientes con discapacidad intelectual inexplicada, retardo del desarrollo psicomotor, problemas del lenguaje, autismo y anomalías congénitas múltiples.

\section{PALABRAS CLAVE}

Cromosomas Humanos par 8; Discapacidad Intelectual; Hibridación Genómica Comparativa; Hipotonía; Trastornos de Ios Cromosomas

\section{SUMMARY}

\section{Cohen syndrome diagnosed using microarray comparative genomic hibridization}

Cohen syndrome (CS) is an uncommon autosomal recessive genetic disorder attributed to damage on VPS13B gene, locus 8q22-q23. Characteristic phenotype consists of intellectual

1 Profesor de los departamentos de Morfología y Ginecoobstetricia de la Univerisdad del Valle, Cali, Colombia.

2 Estudiante de Medicina y Cirugía, Universidad del Valle, Cali, Colombia.

3 Profesor del Departamento de Morfología, Universidad del Valle, Cali, Colombia.

Correspondencia:Wilmar Saldarriaga-Gil; wilmar.saldarriaga@correounivalle.edu.co

Recibido: mayo 31 de 2016

Aceptado: agosto 15 de 2016

Cómo citar: Saldarriaga-Gil W, Collazos-Saa L, Ramírez-Cheyne J. Síndrome de Cohen diagnosticado con hibridación genómica comparativa por microarreglos. latreia. 2017 0ctDic;30(4):455-462. D0I 10.17533/udea.iatreia.v30n4a10. 
disability, microcephaly, facial dysmorphism, ophthalmic abnormalities, truncal obesity and hipotony. Worldwide, around 150 cases have been published, mostly in Finish patients. We report the case of a 3 year-old male, with short height, craniosynostosis, facial dysmorphism, hipotony, and developmental delay. He was diagnosed with Cohen syndrome using Microarray Comparative Genomic Hibridization (aCGH) that showed homozygous deletion of $0.153 \mathrm{Mb}$ on 8q22.2 including VPS13B gene, OMIM \#216550. With this report we contribute to enlarge epidemiological databases on an uncommon genetic disorder. Besides, we illustrate on the contribution of aCGH to the etiological diagnosis of patients with unexplained intellectual disability, delayed psychomotor development, language difficulties, autism and multiple congenital anomalies.

\section{KEY WORDS}

Chromosome Disorders; Comparative Genomic Hybridization; Chromosomes, Human, Pair 8; Hipotony; Intellectual Disability

\section{RESUMO}

\section{Síndrome de Cohen diagnosticado com hibridação genômica comparativa por microarranjos}

A síndrome de Cohen (SC) é uma doenças genética rara, com herança autossômica recessiva. Se origina por danos no gene VPS13B, locus 8q22-q23. O fenótipo característico consiste em deficiência intelectual, microcefalia, faces dismórfica, anormalidades oftalmológicas, obesidade central e hipotonia. Só se há publicado aproximadamente 150 casos, a maioria de origem finlandês. Reportamos o caso de um pré-escolar com estatura baixa, craniossinostose, faces dismórfica, hipotonia e retraso do desenvolvimento psicomotor a quem se the diagnosticou SC por meio de Hibridação Genômica Comparativa por microarranjos (HGCm), que mostrou uma deleção em homozigoto de $0.153 \mathrm{Mb}$ em 8q22.2 incluindo o gene VPS13B, OMIM \#216550. Com esta publicação contribuímos ao conhecimento epidemiológico de uma síndrome genética infrequente, e mostramos o aporte da HGCm ao diagnóstico etiológico de pacientes com deficiência intelectual inexplicada, atraso do desenvolvimento psicomotor, problemas da linguagem, autismo e anomalias congénitas múltiplas.

\section{PALAVRAS CHAVE}

Cromossomas Humanos par 8; Deficiência Intelectual; Hibridação Genômica Comparativa; Hipotonia; Transtornos dos Cromossomas.

\section{INTRODUCCIÓN}

El síndrome de Cohen (SC) es una enfermedad genética con herencia autosómica recesiva originada por daños en el gen VPS13B (Vacuolar Protein Sorting 13 Homolog B). Se han descrito mutaciones puntuales, deleciones y duplicaciones del locus 8q22-q23 que incluyen este gen y se traducen en alteraciones de una proteína transmembranal de función desconocida (1). El fenotipo característico consiste en discapacidad intelectual, microcefalia, facies característica, anormalidades oftálmicas, obesidad central e hipotonía $(2,3)$. Es un síndrome infrecuente, informado por primera vez en 1973 por Cohen y colaboradores quienes describieron el fenotipo original. Se han publicado alrededor de 150 casos, la mayoría en pacientes de origen finlandés, pero también se han reportado casos en Cuba y Brasil (4-7).

El objetivo de esta publicación es presentar un nuevo caso de SC y contribuir a la información epidemiológica sobre este trastorno genético infrecuente, específicamente en Colombia y Latinoamérica, diagnosticado por hibridación genómica comparativa por microarreglos, que mostró una deleción en homocigosis de $0.153 \mathrm{Mb}$ en 8q22.2 incluyendo el gen $\mathrm{COH} 1$, OMIM (por la sigla en inglés de Online Mendelian Inheritance in Man) \#216550.

\section{REPORTE DEL CASO}

Paciente de sexo masculino de 3 años y 10 meses en el momento de la consulta, hijo de padres con relación familiar lejana (figura 1).

En el momento del embarazo la madre tenía 42 años, con antecedentes obstétricos de G3 P1 C1; 


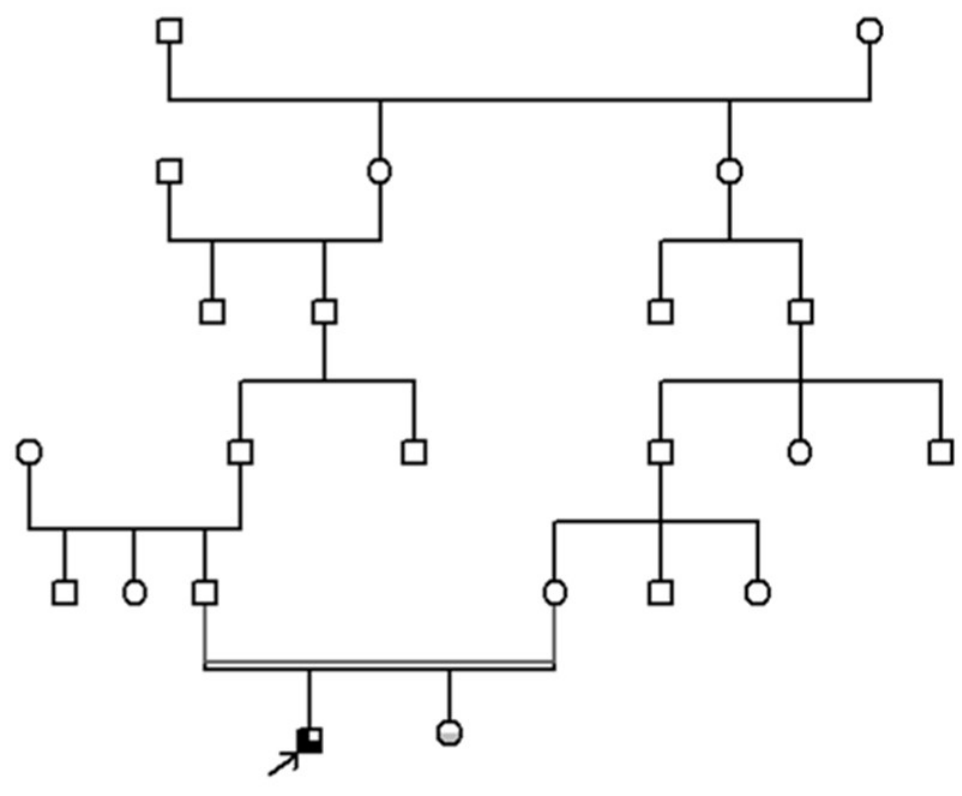

Figura 1. Genealogía. Se observa que el caso aquí reportado es hijo de una unión con parentesco lejano

el primer hijo presentó muerte neonatal temprana de causa desconocida; el segundo es una mujer con discapacidad intelectual, facies dismórfica con dientes centrales prominentes y talla baja, hallazgos sugestivos de SC.

En el embarazo del paciente aquí reportado se hizo control prenatal adecuado con cinco ecografías obstétricas que no detectaron anormalidades; por la edad materna se solicitó cariotipo, que no se hizo por decisión de los padres. Nació por cesárea a las 39 semanas por presentación pođálica; peso 2600 gramos (-1.65 DS), talla $42 \mathrm{~cm}(-4.16 \mathrm{DS})$; observaron deformidad craneal e hicieron tomografía (TAC) que no detectó alteración cerebral; fue dado de alta al día siguiente. A los 3 meses le encontraron hipotonía e iniciaron terapias; a los 8 meses, en una nueva TAC de cráneo, detectaron cierre parcial de las suturas, aumento del diámetro transversal y disminución del anteroposterior, pero sin anomalías cerebrales; se le hizo craneotomía a los 13 meses. En un ecocardiograma a los 3 años no se observaron alteraciones anatómicas. Caminó a los 2,5 años; en el momento de la evaluación solo dice cinco palabras y emite ruidos para comunicarse; no controla esfínteres; los potenciales auditivos son normales, presenta miopía.

Hallazosos relevantes en el examen físico: perímetro cefálico $45 \mathrm{~cm}(<\mathrm{P} 3)$; talla $87 \mathrm{~cm}(-3.59 \mathrm{DS})$; peso $12 \mathrm{~kg}$ (-2.25 DS); diámetro intercantal externo (DICE) $7,8 \mathrm{~cm}$ (+1 DS); diámetro intercantal interno (DICI) $2,8 \mathrm{~cm}$ (0 DS); diámetro interpupilar (IP) $4,5 \mathrm{~cm}(-2$ DS). Facies dismórfica caracterizada por depresión bitemporal, fisuras palpebrales estrechas ligeramente inclinadas hacia abajo, pliegue epicántico marcado en el ojo izquierdo, escleróticas grisáceas (figura 2), incisivos prominentes y micrognatia. Hipotonía marcada, obesidad central insinuada, hipoplasia del pezón derecho, cifoescoliosis, sinostosis radioulnar izquierda, clinodactilia bilateral del quinto dedo (fiơura 3). La impresión diagnóstica fue de síndrome dismórfico de probable origen genético. Se hizo hibridación genómica comparativa por microarreglos (HGCm) que mostró una deleción en homocigosis de $0.153 \mathrm{Mb}$ en 8q22.2 que compromete el gen VPS13B y ha sido relacionada con el síndrome de Cohen, OMIM \#216550. Se hizo dicho diagnóstico por microdeleción (figura 4). 


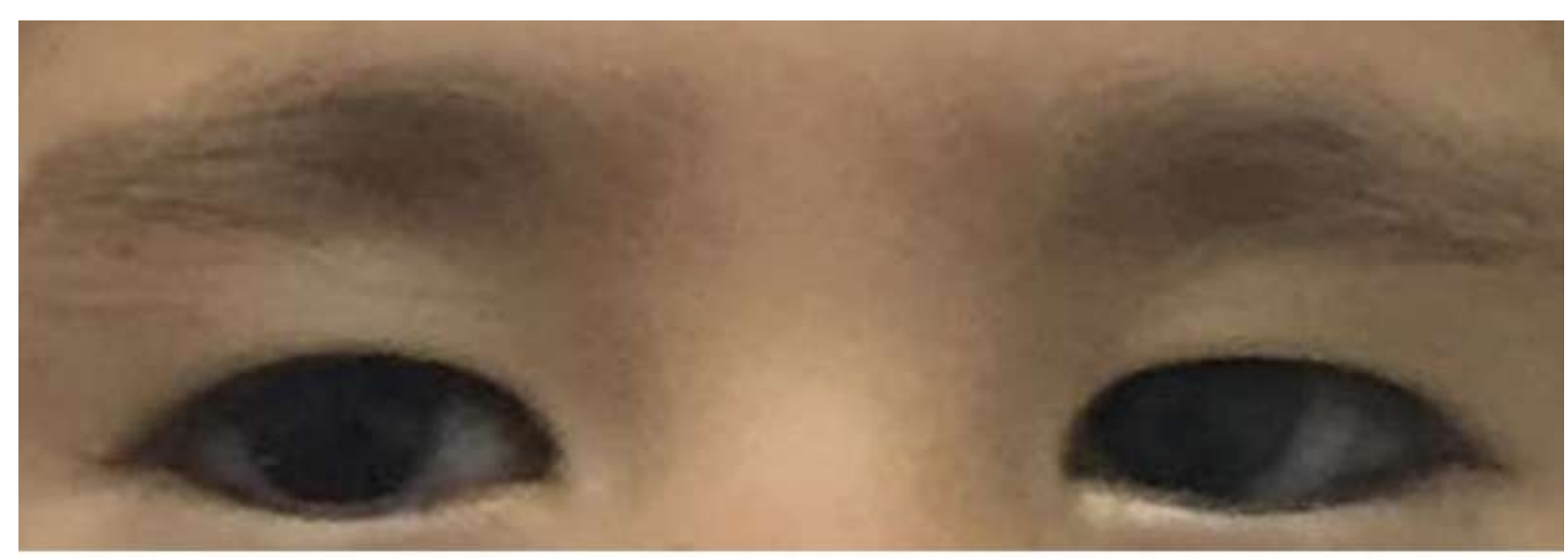

Figura 2. Región periorbitaria. Se observan fisuras palpebrales estrechas ligeramente inclinadas hacia abajo, pliegue epicántico bilateral más marcado en el ojo izquierdo, escleróticas grisáceas y estrabismo

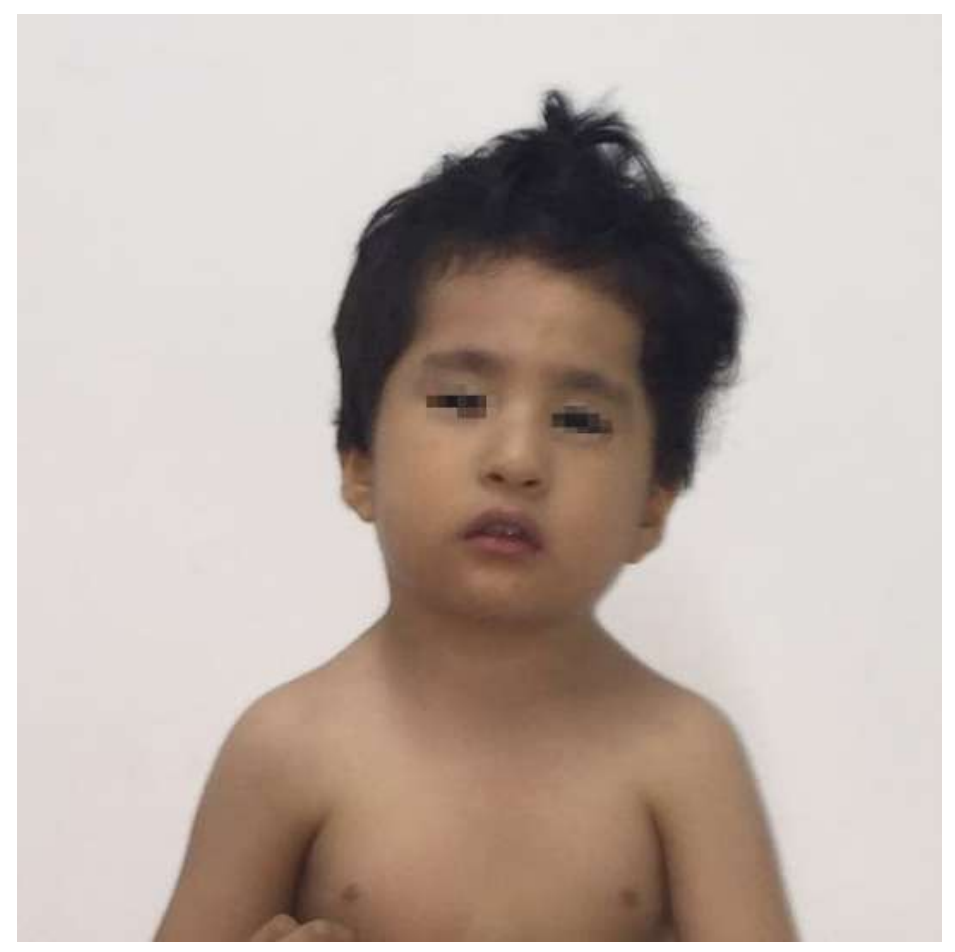

Figura 3. Cara y porción superior del tórax. Se observan signos de hipotonía como la boca abierta y la cabeza inclinada a la izquierda; además, hipoplasia del pezón derecho e incisivos centrales prominentes 


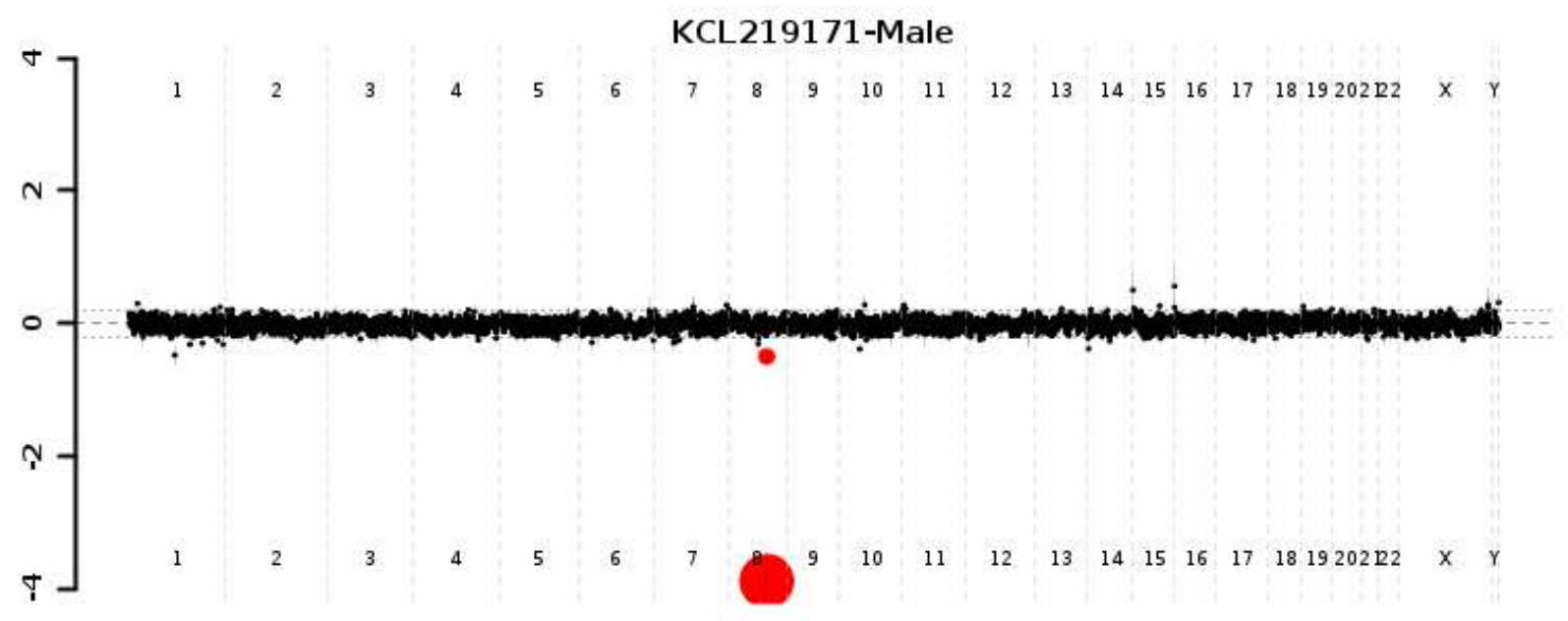

Figura 4. Gráfica del CGHm. En el carril del cromosoma 8 se muestra en los dos puntos de mayor tamaño la deleción en el locus 8q22-q23 de 0.153 Mb que incluye el gen VP13B, criterio diagnóstico de SC.

Los dos padres firmaron el consentimiento informado para tomar fotografías y utilizar los datos de la historia clínica.

\section{DISCUSIÓN}

En 1973, Cohen y colaboradores (7) describieron el síndrome que lleva su nombre en dos hermanos y en un tercer paciente, todos con hipotonía, obesidad, incisivos prominentes y retardo mental; posteriormente, Norio y colaboradores (8) ampliaron la descripción de los siognos a cinco criterios: retardo mental no progresivo acompañado de torpeza motora y microcefalia; rasgos faciales característicos; hipotonía y/o hiperlaxitud articular en la niñez; distrofia retinocoroidea y miopía; neutropenia aislada. Otros rasgos dismórficos que se observan con frecuencia son fisuras palpebrales inclinadas hacia abajo, nariz prominente, surco subnasal (filtrum) corto, boca persistentemente abierta, extremidades delgadas acompañadas de obesidad central en la niñez y comportamiento excesivamente sociable $(2,3)$.

EI SC es una enfermedad genética rara; no existen estudios poblacionales de prevalencia; en la literatura solo hay alrededor de 150 casos informados en una amplia variedad de grupos étnicos y se propone que es un síndrome subdiagnosticado $(4,9)$; la mayoría de estos pacientes son de origen finlandés $y$ muestran un fenotipo homogéneo (10). Se han publicado series de casos en Bélogica, Líbano, Dinamarca e Inglaterra; en grupos humanos aislados como los Amish hay sobrerrepresentación de la enfermedad con un fenotipo muy similar al informado inicialmente en Finlandia, lo que muestra el carácter recesivo de la enfermedad. Sin embaroo, el fenotipo, especialmente los rasogos faciales, varía en otros grupos étnicos, lo que dificulta el diagnóstico basado únicamente en los criterios clínicos (11). En Cuba y Brasil se han informado casos de SC con diagnóstico clínico $(5,6)$. El caso aquí publicado sería el primero con diagnóstico molecular en Colombia y Latinoamérica.

EI SC es una enfermedad de origen genético dada por daños en el gen VPS13B, antes denominado COH1, en el locus 8q22-23 (10), que producen cambios en la proteína de su mismo nombre; por homología con modelos en levaduras se le atribuyen funciones relacionadas con el transporte intra- e intercelular de proteínas mediado por vesículas. Estudios funcionales 
mostraron que la proteína VPS13B se localiza en el aparato de Golgi con la proteína de matriz GM130 y es necesaria para el mantenimiento, el ensamble de las cisternas y la regulación de la formación de túbulos de membrana. La ausencia o alteración de esta proteína puede llevar a la disrupción de la integridad del aparato de Golgi y de su funcionalidad; de igual manera, se han asociado las fallas en esta proteína a defectos de glicosilación y a daños en el mantenimiento de la vía lisosomal-endosomal alterando procesos de degradación intracelular (12). No está claro cómo se relacionan los defectos en la proteína VPS13B con los rasgos fenotípicos. Las diferencias en los fenotipos de los pacientes pueden atribuirse a distintas mutaciones en el gen o a las diferencias genéticas entre las poblaciones (13).

Mochida y colaboradores (14) describieron en 2004 una familia francesa con una mutación que cambiaba el marco de lectura y solo truncaba la región C terminal de la proteína dando lugar a una pérdida parcial en la función de VPS13B por una disrupción de la localización subcelular; este hallazgo se hizo en afectados por SC sin microcefalia. Además, se han descrito mutaciones específicas del gen relacionadas con trastornos del espectro autista y con discapacidad intelectual (12).

Dado que en el SC se han documentado diferentes etiologías genéticas como mutaciones puntuales, microdeleciones y microduplicaciones en homocigosis, heterocigosis y en combinación en el gen VPS13B, para el diagnóstico en los pacientes que cumplen con los criterios clínicos de SC $(2,3,8)$ se han propuesto diferentes técnicas moleculares (15).

El Chehadeh y colaboradores (15) en 2011 analizaron las publicaciones en que se describen diferentes técnicas moleculares para el diagnóstico de SC como MLPA (por la siğla en inglés de MuItiplex Iigation-dependent probe amplification), secuenciación del gen e hibridación genómica comparativa por microarreglos de alta resolución (HGCa); concluyeron que al menos en un tercio de las familias en las que se encuentra una causa genética del SC se observan variaciones en el número de copias de oligonucleótidos que incluyen el gen VPS13B y que se pueden detectar mediante HGCa de alta resolución (244K) y que no necesariamente se deben tener sondas específicas. Además, afirmaron que el MLPA con sondas específicas para
VPS13B es eficiente para el diagnóstico de SC, pero solamente se hace en muy pocos laboratorios por la baja prevalencia del SC. Propusieron que la primera prueba que se haga en pacientes con sospecha clínica de SC sea la HCGa de alta resolución por la facilidad del acceso a ella en todo el mundo. El diagnóstico del paciente aquí reportado se hizo con dicha prueba y se encontró una deleción de $0.153 \mathrm{Mb}$ que incluía el gen VP13B (16).

La HGCm se ha convertido en una herramienta fundamental para el diagnóstico etiológico en individuos con discapacidad intelectual inexplicada, retardo del desarrollo psicomotor, problemas del lenguaje, autismo y anomalías congénitas múltiples; supera las probabilidades de diagnóstico de las pruebas convencionales de citogenética y es fundamental para el enfoque de casos con sospecha de enfermedades de origen genético sin diagnóstico evidente (17). Los individuos afectados por el SC cumplen con estas características especialmente antes de los 5 años.

En el diagnóstico diferencial del SC se deben tener en cuenta los síndromes de Barnet-Biedl y Alström que cursan con retardo mental, retinopatía y obesidad; sin embarogo, la retinopatía en el SC causa una pérdida de la visión periférica, a diferencia de los síndromes mencionados en los que dicha pérdida es de la visión central. Además, en el síndrome de BarnetBiedl se presentan polidactilia postaxial $y$ displasia renal, $y$ en el de Alström los pacientes generalmente tienen el intelecto normal, sordera, cardiomiopatía y diabetes mellitus; estas características no las tienen los afectados por SC.

El síndrome de glicoproteínas deficientes en carbohidratos tipo 1 es otra enfermedad en la que se presentan retraso del desarrollo, hipotonía, microcefalia, obesidad central y retinopatía piomentaria; el diagnóstico diferencial con el SC se basa en estudios de imágenes e inmunoensayos en los que la atrofia olivopontocerebelosa y ensayos de actividad de la fosfomanomutasa logran establecer un defecto en el metabolismo de las glicoproteínas (2).

No existe un tratamiento específico para los pacientes con SC; dado que tienen bajo peso al nacer, problemas con la alimentación, hipotonía durante la infancia, dificultades para alcanzar los hitos del desarrollo y especialmente retraso en el lenguaje, requieren terapia física, ocupacional y del lenguaje; además, 
asistir a escuelas especiales donde se pueda atender a sus necesidades educativas. La miopía progresiva y la retinopatía piomentaria hacen necesarias intervenciones quirúrgicas por el oftalmólogo (2).

La consejería genética para los padres de los niños afectados por SC dependerá del daño en el gen que ocasionó el fenotipo; dado que la mayoría de los casos son por herencia autosómica recesiva, la probabilidad de tener un segundo hijo afectado es de 25 $\%$. En términos generales será ideal para los progenitores hacerse pruebas moleculares para ratificar la sospecha de que son portadores de la mutación en heterocigosis. En el caso aquí reportado los padres tenían una relación lejana de consanguinidad y para el momento de la evaluación no tenían expectativa reproductiva, por lo que no se solicitaron pruebas específicas como FISH (por la sigla en inglés de Fluorescence in situ hybridization) de la región 8q22-23.

\section{CONCLUSIONES}

Reportamos el caso de un infante con diagnóstico molecular de SC logrado mediante $\mathrm{HGCm}$, en la que se encontró una deleción en homocigosis de 0.153 Mb en el locus 8q22.2 incluyendo el gen COH1, OMIM \#216550. Este es el primer caso con diagnóstico molecular reportado en la literatura revisada en Colombia y Latinoamérica. Además, se muestra el aporte de la $\mathrm{HGCm}$ para el diagnóstico etiológico en casos de discapacidad intelectual inexplicada, retardo en el desarrollo psicomotor, problemas del lenguaje, autismo y anomalías congénitas múltiples.

\section{CONFLICTOS DE INTERESES}

Ninguno por declarar

\section{REFERENCIAS BIBLIOGRÁFICAS}

1. Bugiani M, Gyftodimou Y, Tsimpouka P, Lamantea E, Katzaki E, d'Adamo P, et al. Cohen syndrome resulting from a novel large intragenic $\mathrm{COH} 1$ deletion segregating in an isolated Greek island population. Am J Med Genet A. 2008 Sep;146A(17):2221-6. DOI 10.1002/ajmg.a.32239.
2. Chandler KE, Kidd A, Al-Gazali L, Kolehmainen J, Lehesjoki AE, Black GC, et al. Diagnostic criteria, clinical characteristics, and natural history of Cohen syndrome. J Med Genet. 2003 Apr;40(4):233-41.

3. Kolehmainen J, Wilkinson R, Lehesjoki AE, Chandler K, Kivitie-Kallio S, Clayton-Smith J, et al. Delineation of Cohen syndrome following a large-scale genotype-phenotype screen. Am J Hum Genet. 2004 JuI;75(1):122-7.

4. Aitken KJ. Cohen syndrome. In: An A-Z of genetic factors in autism: a handbook for professionals. London: Jessica Kingsley; 2010. p. 164-5.

5. Rim PH, Fiqueirêdo ES, Hirata FE, Steiner CE, Marques-de-Faria AP. Ocular findings in Brazilian identical twins with Cohen syndrome: case report. Arq Bras Oftalmol. 2009 Nov-Dec;72(6):815-8.

6. Santana Hernández EE, Tamayo Chang VJ. Síndrome de Cohen. Presentación de un caso. Medisur. 2014 Abr; 12(2):437-41.

7. Cohen MM Jr, Hall BD, Smith DW, Graham CB, Lampert KJ. A new syndrome with hypotonia, obesity, mental deficiency, and facial, oral, ocular, and limb anomalies. J Pediatr. 1973 Aug;83(2):280-4.

8. Norio R, Raitta C, Lindahl E. Further delineation of the Cohen syndrome; report on chorioretinal dystrophy, leukopenia and consanguinity. Clin Genet. 1984 Jan;25(1):1-14.

9. Wang H, Falk MJ, Wensel C, Traboulsi EI. Cohen Syndrome. In: Pagon RA, Adam MP, Ardinger HH, Wallace SE, Amemiya A, Bean LJH, et al, editors. GeneReviews ${ }^{\circledR}$ [Internet]. Seattle (WA): University of Washington, Seattle; 1993-2017. Available from http:// www.ncbi.nIm.nih.gov/books/NBK1482/

10. Kolehmainen J, Black GC, Saarinen A, Chandler K, Clayton-Smith J, Träskelin AL, et al. Cohen syndrome is caused by mutations in a novel gene, $\mathrm{COH} 1$, encoding a transmembrane protein with a presumed role in vesicle-mediated sorting and intracellular protein transport. Am J Hum Genet. 2003 Jun; 72(6): 1359-69.

11. Falk MJ, Feiler HS, Neilson DE, Maxwell K, Lee JV, Segall SK, et al. Cohen syndrome in the Ohio Amish. Am J Med Genet A. 2004 JuI;128A(1):23-8.

12. Rafiq MA, Leblond CS, Saqib MA, Vincent AK, Ambalavanan A, Khan FS, et al. Novel VPS13B Mutations in Three Large Pakistani Cohen Syndrome Families Suggests a Baloch Variant with Autistic-Like 
Features. BMC Med Genet. 2015 Jun 25;16:41. DOI 10.1186/s12881-015-0183-0.

13. Horn D, Krebsová A, Kunze J, Reis A. Homozygosity mapping in a family with microcephaly, mental retardation, and short stature to a Cohen syndrome region on 8q21.3-8q22.1: redefining a clinical entity. Am J Med Genet. 2000 Jun;92(4):285-92.

14. Mochida GH, Rajab A, Eyaid W, Lu A, AI-Nouri D, Kosaki K, et al. Broader geographical spectrum of Cohen syndrome due to $\mathrm{COH} 1$ mutations. J Med Genet. 2004 Jun;41(6):e87.

15. El Chehadeh-Djebbar S, Faiøre L, Moncla A, Aral B, Missirian C, Popovici C, et al. The power of high-resolution non-targeted array-CGH in identifying intragenic rearrangements responsible for Cohen syndrome. J Med Genet. 2011 Nor;48(11):e1. DOI 10.1136/jmg.2011.088948.

16. Rivera-Brugués N, Albrecht B, Wieczorek D, Schmidt H, Keller T, Göhring I, et al. Cohen syndrome diagnosis using whole genome arrays. J Med Genet. 2011 Feb;48(2):136-40. DOI 10.1136/jmg.2010.082206.

17. Ruiz Botero F, Saldarriaga Gil W, Isaza de Lourido C. [Chromosome 7q11.23 duplication syndrome. First reported case in Latin America]. Arch Aroent pediatr. 2016 Feb;114(1):e1-4. DOI 10.5546/aap.2016. e1. Spanish. 